\title{
Inverse Analysis for Estimating Damage Patterns in Notched Composite Laminates Using an Embedded FBG Sensor*
}

\author{
Shigeki YASHIRO $^{* *}$ and Nobuo TAKEDA ${ }^{* * *}$ \\ ${ }^{* *}$ Research Institute of Instrumentation Frontier, National Institute of Advanced Industrial Science and \\ Technology (AIST), \\ 1-1-1 Umezono, Tsukuba, Ibaraki 305-8568, Japan \\ E-mail: yashiro-s@aist.go.jp \\ ${ }^{* * *}$ Graduate School of Frontier Sciences, The University of Tokyo, \\ 5-1-5 Kashiwanoha, Kashiwa, Chiba 277-8561, Japan \\ E-mail: takeda@smart.k.u-tokyo.ac.jp
}

\begin{abstract}
This study develops a new inverse analysis technique for estimating damage patterns in notched composite laminates using an embedded fiber Bragg grating (FBG) sensor. The damage pattern near the notch was investigated by using a layer-wise finite-element model with cohesive elements for cracks and delamination. The reflection spectrum of the FBG sensor was simulated from the strain distribution obtained along the gage length. We approximated the typical damage pattern (splits, transverse cracks, and delamination) near the notch using some variables; these were then estimated through mathematical programming by minimizing the differences of spectrum shapes between the inputs (e.g. experiments) and the estimations. This damage identification procedure was applied to numerical examples, i.e. simulation results, for a notched CFRP cross-ply laminate. The damage patterns estimated from the calculated reflection spectra were almost identical to those obtained by the simulation. Finally, we estimated the damage pattern of a notched laminate from a measured reflection spectrum of an embedded FBG sensor and demonstrated the capability of the developed identification procedure for practical applications.
\end{abstract}

Key words: Composite Material, Finite Element Method, Inverse Problem, Notch, Delamination, FBG Sensor

\section{Introduction}

Structural health monitoring has recently been recognized as a key technology to ensure the safety of structures with a long operational lifetime such as buildings, plants and aircraft $^{(1)-(8)}$. Fiber Bragg grating (FBG) sensors have attracted a lot of attention in health monitoring applications due to their accurate measurement for strain and/or temperature and multiplexing capability ${ }^{(9)(10)}$. Furthermore, FBG sensors have a high sensitivity to strain distribution; a non-uniform distribution of the longitudinal strain causes a large deformation in the spectrum for the light reflected from the gage section ${ }^{(10)}$. Takeda and his colleagues ${ }^{(11)(12)}$ detected damage, i.e. transverse cracks and delamination, in composite laminates using this advantage of FBG sensors.

Complex damage patterns can often be generated due to the stress concentration in composite laminates. Therefore, extensive experimental and analytical studies ${ }^{(13)-(15)}$ have

*Received 20 Dec., 2006 (No. T-04-1232) Japanese Original : Trans. Jpn. Soc. Mech. Eng., Vol. 71, No. 706, A (2005), pp.897-904 (Received 18 Nov., 2004) [DOI: 10.1299/jmmp.1.310] 
been performed to clarify the damage process. Kortschot ${ }^{(13)}$ demonstrated that a notch induced a characteristic damage pattern that concurrently included splits, transverse cracks and delamination. The authors ${ }^{(16)}$ studied damage-monitoring for a notched CFRP laminate using an embedded FBG sensor and demonstrated that its reflection spectrum contained considerable information about the damage pattern through the longitudinal strain distribution. This result suggested that the damage pattern in a laminate could be identified by analyzing the reflection spectrum of the embedded FBG sensor.

The purpose of this study is then to identify the damage in a notched composite laminate using the embedded FBG sensor. For this purpose, we developed an inverse analysis for estimating damage patterns, i.e. types of damage, their locations and sizes, from the reflection spectrum. Section 2 describes a combined damage- (structural-) optical analysis to simulate a reflection spectrum of the FBG sensor embedded in a laminate ${ }^{(16)}$. Section 3 then develops a damage identification procedure that mathematically estimates a damage pattern using a spectrum shape of the embedded FBG sensor. Finally, in section 4, we applied the damage identification to numerical examples and an experimental result for a notched CFRP cross-ply laminate, and discuss these results.

\section{Analysis}

\subsection{Damage analysis}

The damage process in a notched CFRP cross-ply laminate was simulated using a layer-wise finite-element model with cohesive elements ${ }^{(16)}$. Figure 1 illustrates the finite-element mesh considering the symmetry. The dimensions were $15 \mathrm{~mm}$ in the longitudinal $(x)$ direction and $10.5 \mathrm{~mm}$ in the transverse $(y)$ direction, and the notch was 4.5 $\mathrm{mm}$ long. The model was separated into layers of $0^{\circ}$ and $90^{\circ}$ plies to express the stacking configuration of $\left[0_{2} / 90_{2}\right]_{\mathrm{s}}$. Both layers were $0.25 \mathrm{~mm}$ thick, and four-node Mindlin plate elements were applied to these layers to address out-of-plane deformation. Here, plate elements were used to reduce the computation costs from 3-D solid elements. An optical fiber was built into the $0^{\circ}$ layer as two-node truss elements along the $x$-direction $1.8 \mathrm{~mm}$ from the notch tip.

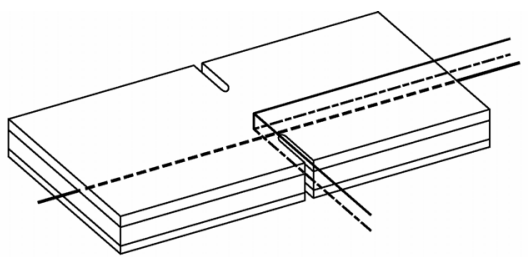

(a) Schematic

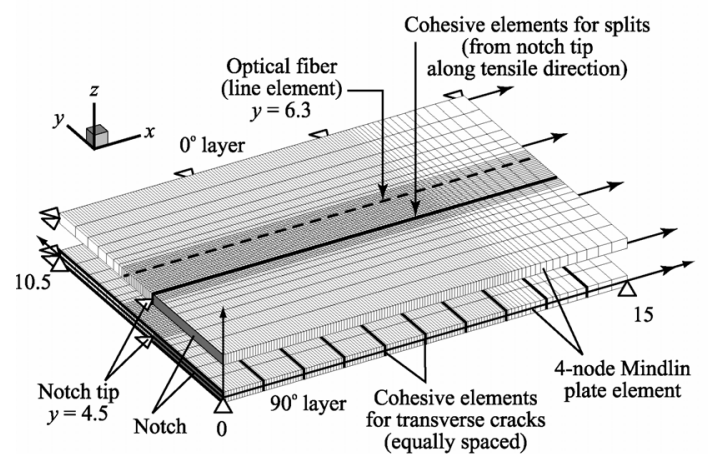

(b) Finite-element mesh

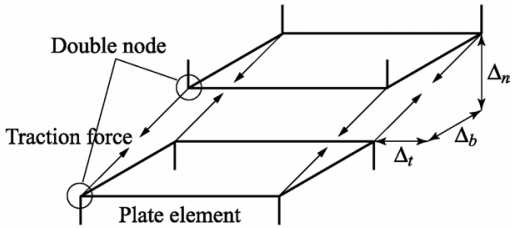

(c) Cohesive element for delamination

Fig. 1 Layer-wise finite-element model of the notched cross-ply laminate with an embedded optical fiber. Cohesive elements for delamination are inserted into all $0^{\circ} / 90^{\circ}$ layer interfaces. 
This damage analysis used cohesive elements to express splits, transverse cracks (four-node elements), and delamination (eight-node elements) based on the experiment ${ }^{(16)}$. Cohesive elements were assigned to the boundaries between the two plate elements where cracks could be initiated, as depicted in Fig. 1. These cohesive elements act as nonlinear springs linking the plate elements and generate traction resisting the relative displacement between them.

Equation (1) expresses the principle of virtual work for the layer-wise model.

$$
\int_{V_{p}} \delta \varepsilon_{p}: \sigma_{p} \mathrm{~d} V+\int_{V_{f}} \delta \boldsymbol{\varepsilon}_{f}: \sigma_{f} \mathrm{~d} V+\int_{S_{c o h}} \delta \boldsymbol{\Delta} \cdot \boldsymbol{T} \mathrm{d} S=\int_{S_{c o h}} \delta \boldsymbol{u} \cdot \boldsymbol{t}_{e x} \mathrm{~d} S
$$

$\sigma$ and $\varepsilon$ denote the stress and strain tensors, while subscripts $p$ and $f$ indicate the laminate and the optical fiber. $\boldsymbol{T}$ and $\boldsymbol{\Delta}$ represent the traction vector and the relative displacement vector in a cohesive element. $\boldsymbol{t}_{e x}$ and $\boldsymbol{u}$ are the external force vector and the displacement vector.

The relation between the traction and the relative displacement is expressed in terms of a residual strength parameter $s^{(17)}$.

$$
\boldsymbol{T}=\left\{\begin{array}{l}
T_{n} \\
T_{t} \\
T_{b}
\end{array}\right\}=\left[\begin{array}{ccc}
\frac{s}{1-s} \frac{\tau_{n \max }}{\Delta_{n c}} & 0 & 0 \\
0 & \frac{s}{1-s} \frac{\tau_{t \max }}{\Delta_{t c}} & 0 \\
0 & 0 & \frac{s}{1-s} \frac{\tau_{b \max }}{\Delta_{b c}}
\end{array}\right]\left\{\begin{array}{l}
\Delta_{n} \\
\Delta_{t} \\
\Delta_{b}
\end{array}\right\}=\boldsymbol{D}_{c o h} \Delta
$$

$T$ and $\Delta$ are the components of the traction and the relative displacement at a given point in a cohesive element. Subscripts $n, t$, and $b$ indicate the deformation modes of normal tensile cracking (mode I), in-plane shear cracking (mode II), and out-of-plane shear cracking (mode III). $\tau_{i \max }(i=n, t, b)$ are the maximum stresses in these cracking modes. The critical relative displacements $\Delta_{i c}(i=n, t, b)$ are defined by the following expression:

$$
\Delta_{n c}=\frac{2 G_{\mathrm{I} c}}{\tau_{n \max } S_{i n i}}, \quad \Delta_{t c}=\frac{2 G_{\mathrm{II} c}}{\tau_{t \max } S_{i n i}}, \quad \Delta_{b c}=\frac{2 G_{\mathrm{III} c}}{\tau_{b \max } s_{i n i}}
$$

where $G_{\text {I } c}, G_{\text {II } c}$ and $G_{\text {IIIc }}$ are the critical energy release rates for each cracking mode, and $s_{\text {ini }}$ $(=0.999)$ is the initial value of the residual strength parameter. The residual strength parameter is defined as a function of the normalized relative displacement vector $\widetilde{\Delta}$.

$$
\begin{aligned}
& s=\min \left[s_{\min }, \max [0,1-|\widetilde{\Delta}|]\right] \\
& \tilde{\Delta}=\left\{\Delta_{n} / \Delta_{n c}, \Delta_{t} / \Delta_{t c}, \Delta_{b} / \Delta_{b c}\right\}^{T}
\end{aligned}
$$

The displacement vector $\boldsymbol{u}=\{u, v, w\}^{T}$ is represented by the displacements $(\hat{u}, \hat{v}$ and $\hat{w})$ and rotation $\left(\theta_{x}\right.$ and $\theta_{y}$ ) on the mid plane of a layer based on the Mindlin plate theory.

$$
\begin{aligned}
u & =\hat{u}(x, y)-z \theta_{x}(x, y) \\
v & =\hat{v}(x, y)-z \theta_{y}(x, y) \\
w & =\hat{w}(x, y)
\end{aligned}
$$

The relative displacement vector $\Delta$ for the delamination is then determined at the layer interface as follows: 


$$
\begin{aligned}
& \Delta=\left\{\begin{array}{l}
\Delta_{n} \\
\Delta_{t} \\
\Delta_{b}
\end{array}\right\}=\left[\begin{array}{cccccccccc}
0 & 0 & -1 & 0 & 0 & 0 & 0 & 1 & 0 & 0 \\
-1 & 0 & 0 & \frac{t_{l}}{2} & 0 & 1 & 0 & 0 & \frac{t_{u}}{2} & 0 \\
0 & -1 & 0 & 0 & \frac{t_{l}}{2} & 0 & 1 & 0 & 0 & \frac{t_{u}}{2}
\end{array}\right]\left\{\begin{array}{c}
\hat{\boldsymbol{u}}_{l} \\
\hat{\boldsymbol{u}}_{u}
\end{array}\right\}=\boldsymbol{L}\left\{\begin{array}{c}
\hat{\boldsymbol{u}}_{l} \\
\hat{\boldsymbol{u}}_{u}
\end{array}\right\} \\
& \hat{\boldsymbol{u}}=\left\{\begin{array}{lllll}
\hat{u} & \hat{v} & \hat{w} & \theta_{x} & \theta_{y}
\end{array}\right\}^{T}
\end{aligned}
$$

where $t$ is the layer thickness, and subscripts $u$ and $l$ indicate the upper and lower layers. The displacement in an element $e$ is interpolated from the nodal displacement $\hat{\boldsymbol{U}}$ using the shape function $N^{i}$ of node $i$.

$$
\begin{aligned}
& \left\{\begin{array}{l}
\hat{\boldsymbol{u}}_{l} \\
\hat{\boldsymbol{u}}_{u}
\end{array}\right\}=\left[\begin{array}{cccccccc}
\boldsymbol{N}^{1} & \boldsymbol{N}^{2} & \boldsymbol{N}^{3} & \boldsymbol{N}^{4} & \mathbf{0} & \mathbf{0} & \mathbf{0} & \mathbf{0} \\
\mathbf{0} & \mathbf{0} & \mathbf{0} & \mathbf{0} & \boldsymbol{N}^{1} & \boldsymbol{N}^{2} & \boldsymbol{N}^{3} & \boldsymbol{N}^{4}
\end{array}\right] \hat{\boldsymbol{U}}_{e}=\boldsymbol{N}_{c o h} \hat{\boldsymbol{U}}_{e} \\
& \boldsymbol{N}^{i}=N^{i} \boldsymbol{I}^{5}, \\
& \boldsymbol{I}^{5} \text { : unit matrix }(5 \times 5) \\
& \hat{\boldsymbol{U}}_{e}=\left\{\begin{array}{llllllll}
\hat{\boldsymbol{U}}_{l}^{1} & \hat{\boldsymbol{U}}_{l}^{2} & \hat{\boldsymbol{U}}_{l}^{3} & \hat{\boldsymbol{U}}_{l}^{4} & \hat{\boldsymbol{U}}_{u}^{1} & \hat{\boldsymbol{U}}_{u}^{2} & \hat{\boldsymbol{U}}_{u}^{3} & \hat{\boldsymbol{U}}_{u}^{4}
\end{array}\right\}^{T}
\end{aligned}
$$

Substituting Eqs. (2) through (7) into Eq. (1) yields the matrix expression for the principle of virtual work.

$$
\begin{aligned}
& \delta \boldsymbol{U}^{T} \boldsymbol{K} \boldsymbol{U}=\delta \boldsymbol{U}^{T} \boldsymbol{f} \\
& \boldsymbol{K}=\boldsymbol{K}_{p}+\boldsymbol{K}_{f}+\boldsymbol{K}_{c o h} \\
& \boldsymbol{K}_{p}=\int_{V_{p}} \boldsymbol{B}_{p}{ }^{T} \boldsymbol{D}_{p} \boldsymbol{B}_{p} \mathrm{~d} V \\
& \boldsymbol{K}_{f}=\int_{V_{f}} \boldsymbol{B}_{f}{ }^{T} \boldsymbol{D}_{f} \boldsymbol{B}_{f} \mathrm{~d} V \\
& \boldsymbol{K}_{c o h}=\int_{S_{c o h}} \boldsymbol{N}_{c o h}{ }^{T} \boldsymbol{L}^{T} \boldsymbol{D}_{c o h} \boldsymbol{L} \boldsymbol{N}_{c o h} \mathrm{~d} S \\
& \boldsymbol{f}=\int_{S_{e x}} \boldsymbol{N}^{T} \boldsymbol{t}_{e x} \mathrm{~d} S+\int_{V_{p}} \boldsymbol{B}_{p}{ }^{T} \boldsymbol{D}_{p} \boldsymbol{\alpha}_{p} \Delta T \mathrm{~d} V+\int_{V_{f}} \boldsymbol{B}_{f}{ }^{T} \boldsymbol{D}_{f} \boldsymbol{\alpha}_{f} \Delta T \mathrm{~d} V
\end{aligned}
$$

where $\boldsymbol{U}$ and $\boldsymbol{f}$ are the nodal displacement vector and the nodal external force vector for the whole model. $\boldsymbol{K}, \boldsymbol{B}$ and $\boldsymbol{D}$ denote the stiffness matrix, the strain-displacement matrix and the constitutive coefficients. Subscripts $p, f$ and coh indicate the plate element with anisotropy for each layer, the truss element for the optical fiber, and the cohesive element. $N$ is the shape function matrix. Thermal residual stresses for the temperature change $\Delta T(=-165 \mathrm{~K})$ are also considered as the nodal external force vector, and $\alpha$ represents thermal expansion coefficients in the vector format. We applied the direct iteration method ${ }^{(18)}$ to converge the solution of the nonlinear equation (8). Material properties used in this analysis are listed in Table 1.

\subsection{Optical analysis}

Figure 2 illustrates the schematic of an FBG sensor. The gage section of the FBG sensor has periodic changes in the refractive index of the core in the optical fiber. A narrow band component is then reflected at the gage section following the incidence of broadband light; the wavelength of the reflection is proportional to the period of the index modulation ${ }^{(9)(10)}$.

The complex amplitudes $A(x)$ and $B(x)$ for the forward and backward light waves, where $x$ is the longitudinal direction of the optical fiber, obey the following coupled-mode equations. 
Table 1 Material properties used in the analysis.

\begin{tabular}{ll}
\hline (a) Material properties & \\
CFRP $(T 800 H / 3631)$ & 148 \\
Longitudinal Young's modulus $(\mathrm{GPa})$ & 9.57 \\
Transverse Young's modulus $(\mathrm{GPa})$ & 4.50 \\
In-plane shear modulus $(\mathrm{GPa})$ & 3.5 \\
Out-of-plane shear modulus $(\mathrm{GPa})$ & 0.356 \\
In-plane Poisson's ratio & 0.49 \\
Out-of-plane Poisson's ratio & -0.6 \\
Longitudinal thermal expansion coefficient $\left(\times 10^{-6} \mathrm{~K}^{-1}\right)$ & 36.0 \\
Transverse thermal expansion coefficient $\left(\times 10^{-6} \mathrm{~K}^{-1}\right)$ & \\
$\quad$ Optical fiber & 73.1 \\
Young's modulus of glass $(\mathrm{GPa})$ & 1.47 \\
Young's modulus of coating $(\mathrm{GPa})$ & 0.5 \\
Thermal expansion coefficient of glass $\left(\times 10^{-6} \mathrm{~K}^{-1}\right)$ & 60 \\
Thermal expansion coefficient of coating $\left(\times 10^{-6} \mathrm{~K}^{-1}\right)$ & 60 \\
\end{tabular}

(b) Properties for cohesive elements

For splits and transverse cracks

In-plane tensile strength (Mode I, MPa)

In-plane shear strength (Mode II, MPa)

Out-of-plane shear strength (Mode III, MPa)

Critical energy release rate (Mode I, $\mathrm{J} / \mathrm{m}^{2}$ )

Critical energy release rate (Mode II, $\mathrm{J} / \mathrm{m}^{2}$ )

Critical energy release rate (Mode III, $\mathrm{J} / \mathrm{m}^{2}$ )

For delamination

In-plane tensile strength (Mode I, MPa)

In-plane shear strength (Mode II, MPa)

Out-of-plane shear strength (Mode III, MPa)

Critical energy release rate (Mode I, $\mathrm{J} / \mathrm{m}^{2}$ )

Critical energy release rate (Mode II, $\mathrm{J} / \mathrm{m}^{2}$ )

Critical energy release rate (Mode III, $\mathrm{J} / \mathrm{m}^{2}$ )
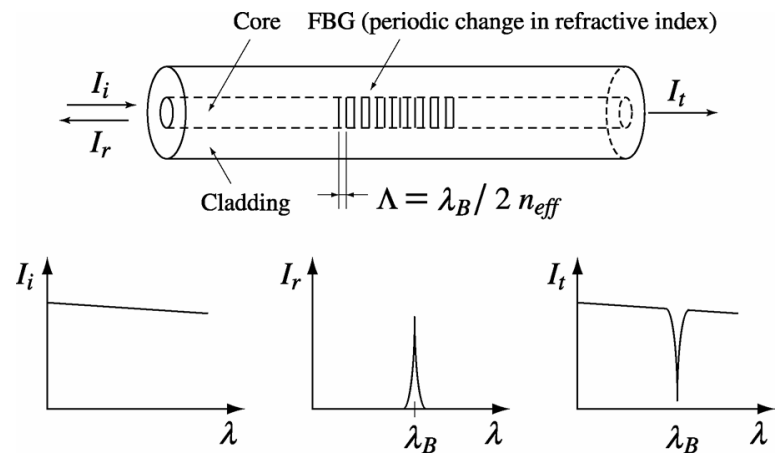

Fig. 2 Schematic of an FBG sensor. When a spectrally broadband light source is injected into the fiber, a narrowband component is reflected at the gage section.

$$
\begin{aligned}
& \frac{\mathrm{d} A}{\mathrm{~d} x}=-i \kappa B \exp [2 i \varphi x] \\
& \frac{\mathrm{d} B}{\mathrm{~d} x}=i \kappa A \exp [-2 i \varphi x]
\end{aligned}
$$

The variables $\kappa$ and $\varphi$ are the mode-coupling coefficient and the parameter detuning to the highest-reflectivity condition. These parameters are expressed in terms of the wavelength $\lambda$ :

$$
\begin{aligned}
& \kappa=\frac{\pi}{\lambda} \delta n \\
& \varphi=\beta-\beta_{0}=\frac{2 \pi}{\lambda} n_{\text {eff }}-\frac{\pi}{\Lambda}
\end{aligned}
$$

where $n_{\text {eff }}$ is the effective refractive index, $\delta n$ is the depth of the refractive index modulation, $\Lambda$ is the grating period, and $\beta_{0}=\pi / \Lambda$. The relationship among $A(0), A(l), B(0)$ and $B(l)$ can be obtained from the general solutions of Eqs. (14) and (15) for a uniform grating within $0 \leq x \leq l$.

$$
\begin{aligned}
& \left\{\begin{array}{l}
A(l) \\
B(l)
\end{array}\right\}=\left[\begin{array}{cc}
a & b \\
b^{*} & a^{*}
\end{array}\right]\left\{\begin{array}{l}
A(0) \\
B(0)
\end{array}\right\} \\
& a=\exp (-i \varphi l)\left[\cosh (\alpha l)-i \frac{\varphi}{\alpha} \sinh (\alpha l)\right] ; \quad b=-i \frac{\kappa}{\alpha} \exp (i \varphi l) \sinh (\alpha l) \\
& a^{*}, b^{*}: \text { conjugates of } a \text { and } b \\
& \alpha=\sqrt{\kappa^{2}-\varphi^{2}}
\end{aligned}
$$


To simulate the reflection spectrum of the FBG sensor, the gratings in a gage section of length $L$ are divided into many segments, and each segment is assumed to have a uniform profile. The mode coupling between the forward wave and the backward wave can then be analyzed by multiplying the transfer matrices in Eq. (18) along the full segments ${ }^{(19)}$.

$$
\begin{aligned}
& \left\{\begin{array}{l}
A(L) \\
B(L)
\end{array}\right\}=\boldsymbol{F}\left\{\begin{array}{l}
A(0) \\
B(0)
\end{array}\right\} \\
& \boldsymbol{F}=\boldsymbol{F}_{M} \boldsymbol{F}_{M-1} \cdots \boldsymbol{F}_{1}, \quad \boldsymbol{F}_{j}=\left[\begin{array}{ll}
a_{j} & b_{j} \\
b_{j}{ }^{*} & a_{j}{ }^{*}
\end{array}\right]
\end{aligned}
$$

We now obtain the reflectivity $R(\lambda)=|B(0)|^{2}$ at a wavelength $\lambda$, under the boundary conditions of $A(0)=1$ and $B(L)=0$.

The grating period and the effective refractive index depend on the longitudinal strain $\varepsilon_{f}(x)$ within the FBG sensor embedded in a laminate ${ }^{(20)}$.

$$
\begin{aligned}
& \Lambda(x)=\left(1+\varepsilon_{f}(x)\right) \Lambda_{i n i} \\
& n_{e f f}(x)=n_{0}+\Delta n(x)=n_{0}-\frac{n_{0}^{3}}{2}\left\{p_{12}-v_{f}\left(p_{11}+p_{12}\right)\right\} \varepsilon_{f}(x)
\end{aligned}
$$

$\Lambda_{i n i}$ is the initial grating period, $n_{0}$ is the initial refractive index of the core, $v_{f}$ is the Poisson's ratio of the glass, and $p_{11}$ and $p_{12}$ denote Pockel constants where indices 1 and 2 indicate the longitudinal and transverse directions of the optical fiber. Substituting the strain distribution of the optical fiber obtained in the damage analysis into Eqs. (21) and (22) and solving Eq. (19) yield a reflection spectrum that contains the effect of the damage in the notched laminate. Table 2 lists the optical properties of the FBG sensor. The gage length of the sensor was $10 \mathrm{~mm}$, and the gage was located at $0 \leq x \leq 10, y=6.3$ in the model (Fig. 1).

The reflection spectra were simulated for the FBG sensor embedded in the notched CFRP cross-ply laminate under tensile loadings. Figures 3 (Simulation) and 4 (Simulation) depict the simulated damage process of the laminate and the corresponding reflection spectra of the FBG sensor. These results agreed well with the experiment results ${ }^{(16)}$.

\section{Development of damage identification}

\subsection{Problem definition}

The grating period has a monotonic distribution along the gage length due to the stress concentration of the notch when an end of the initially uniform FBG sensor is located at the notch tip. Corresponding to the type, location and size of the damage, the position of the grating whose property is changed by generation of damage is then uniquely determined in the reflection spectrum since the wavelength of the reflection is proportional to the grating period. Therefore, the reflection spectrum of the embedded FBG sensor has a one-to-one correspondence to the damage pattern in the laminate. A chirped FBG sensor, which initially has a monotonic distribution in the grating period, should be used to achieve the same condition in the laminate without stress concentration ${ }^{(21)}$.

Table 2 Optical properties of the FBG sensor.

\begin{tabular}{ll}
\hline Gage length $(\mathrm{mm})$ & 10 \\
Initial center wavelength $\lambda_{0}(\mathrm{~nm})$ & 1549.40 \\
Initial refractive index of the core $n_{0}$ & 1.4490 \\
Strain-optic coefficients $p_{11}$ & 0.113 \\
Strain-optic coefficients $p_{12}$ & 0.252 \\
\hline
\end{tabular}


Simulation

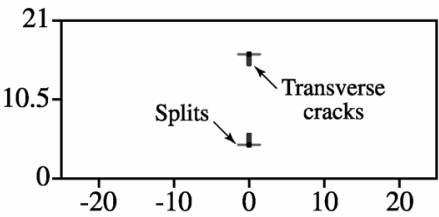

(a) $0.20 \%$

(b) $0.35 \%$

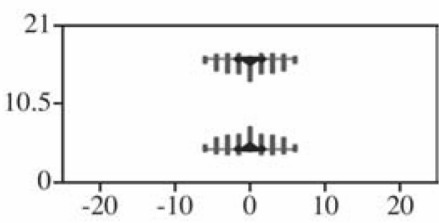

(c) $0.45 \%$

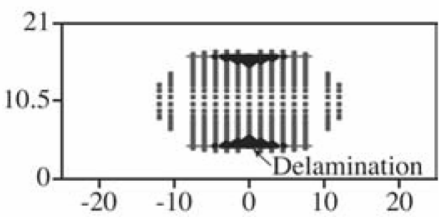

(d) $0.60 \%$

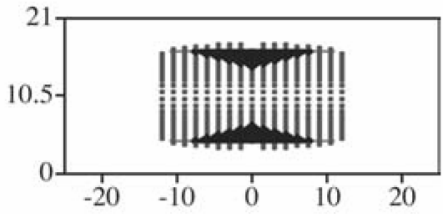

(e) $0.70 \%$

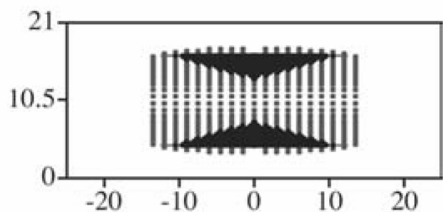

Estimation

(No damage)
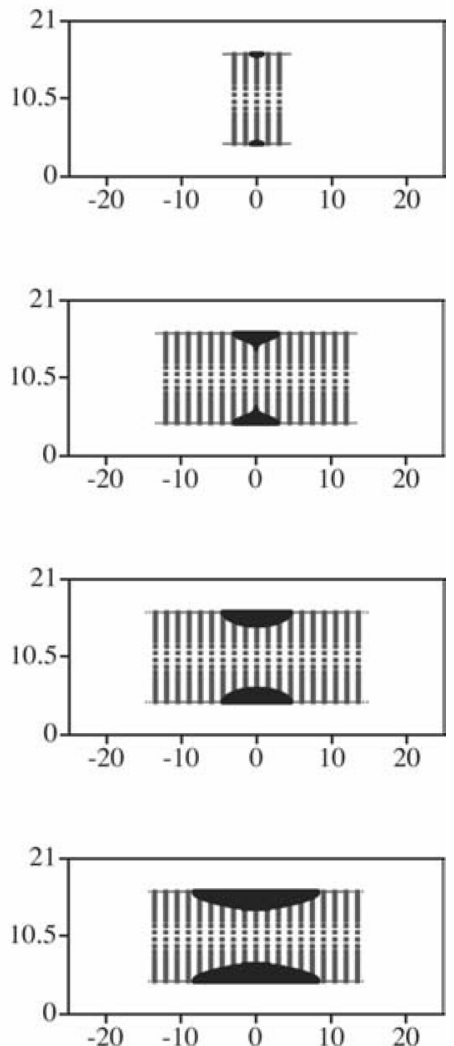

Fig. 3 Damage patterns of the notched cross-ply laminate produced by the simulation and by the estimation. Each dot represents a completely damaged cohesive element.

The damage pattern near the notch was estimated from the shape of the reflection spectrum for the embedded FBG sensor. This problem can be defined as an optimization problem that minimizes the square errors between the input spectrum and the temporarily estimated spectrum as a function of variable $\boldsymbol{d}$ to represent a damage pattern near the notch.

Minimize: $\quad F(\boldsymbol{d})=\sum_{m=0}^{50}\left\{a_{m}-\widetilde{a}_{m}(\boldsymbol{d})\right\}^{2}$

Design variables: $\boldsymbol{d}=\left\{d_{d 1}, d_{d 2}, d_{d 3}, \alpha, \beta, p, d_{c}\right\}^{T}$

The reflection spectra were expressed by a Fourier series under the 50th order to quantitatively evaluate their shapes. Here, $a_{m}$ and $\tilde{a}_{m}$ are the $m$ th Fourier coefficients for the input and estimated spectrum shapes. The variable $\boldsymbol{d}$ that minimizes square errors $F(\boldsymbol{d})$ was considered to be the identified result.

The residual strength parameter $s$ defines the stiffness for each cohesive element in the finite-element model. Therefore, the reflection spectrum $\tilde{a}_{m}$ is optimized by using the change in the strain distribution that results from the stiffness modification in cohesive elements as a function of variable $\boldsymbol{d}$. Figure 5 defines the variables to represent the characteristic damage pattern, i.e. the distribution of the residual strength parameter $s$, near 


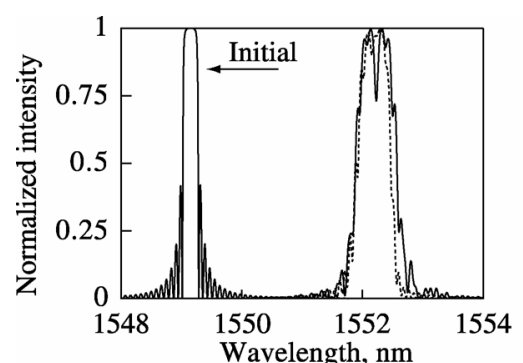

(a) $0.20 \%$

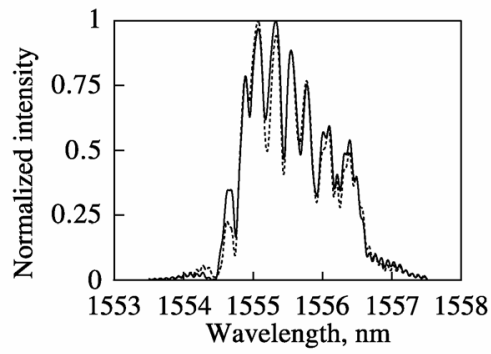

(c) $0.45 \%$

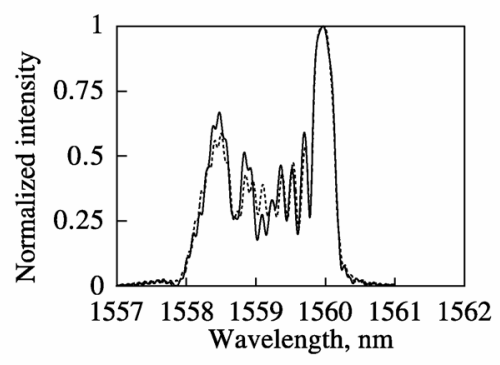

(e) $0.75 \%$

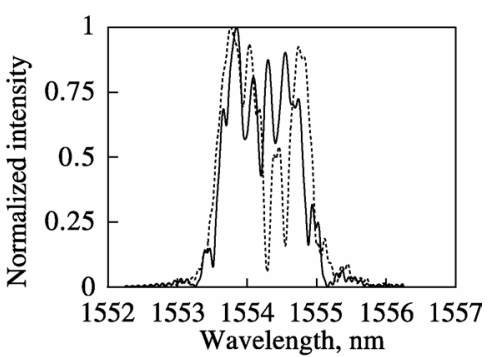

(b) $0.35 \%$

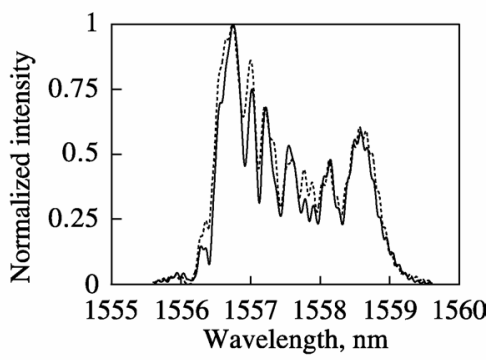

(d) $0.60 \%$

\section{Simulation}

(Input of Eq. (23))

Estimation

Fig. 4 Calculated and estimated reflection spectra (corresponding to Fig. 3).

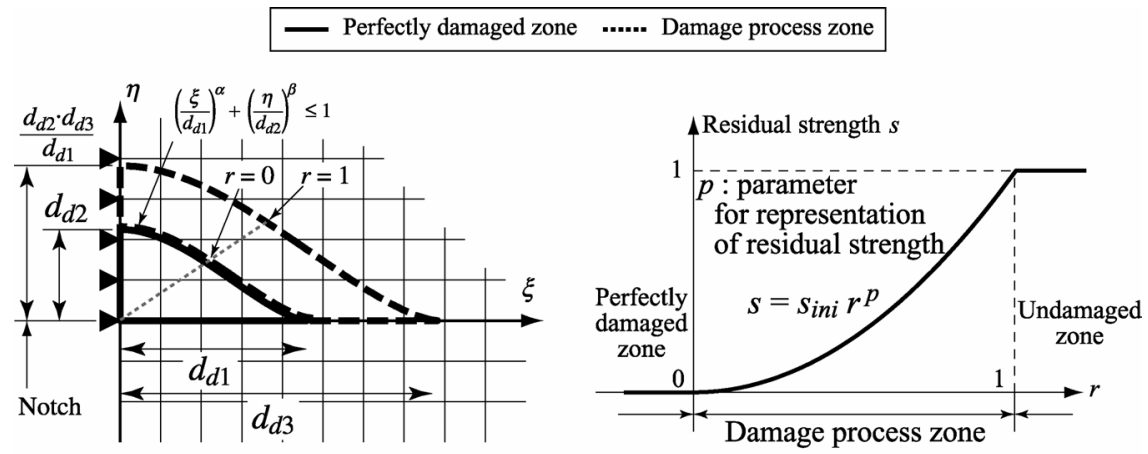

(a) Delamination

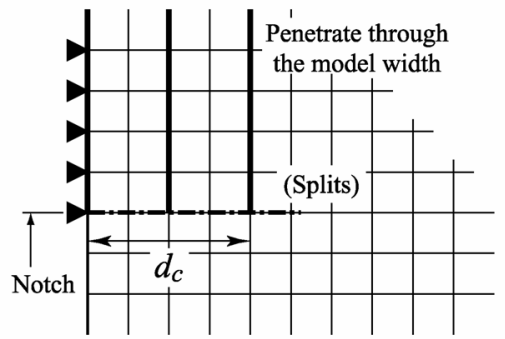

(b) Transverse cracks

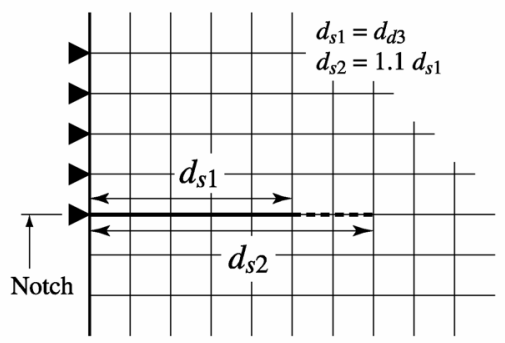

(c) Splits

Fig. 5 Definition of design variables to represent the damage pattern near the notch, i.e. the distribution of the residual strength parameter of the cohesive elements. 
the notch. The delaminated region was approximated by the following equation in the $\xi-\eta$ coordinate system whose origin was located at the notch tip.

$$
\left(\frac{\xi}{d_{d 1}}\right)^{\alpha}+\left(\frac{\eta}{d_{d 2}}\right)^{\beta} \leq 1, \quad \xi \geq 0, \eta \geq 0
$$

The variables $d_{d 1}$ and $d_{d 2}$ express the size; $\alpha$ and $\beta$ define the shape of the delamination. The residual strength parameter $s=0$ was given for each cohesive element satisfying Eq. (24). The delamination process zone (the region where $0<s<s_{\text {ini }}$ ) was also considered, and its size was expressed by the variable $d_{d 3}$. The value $s$ in the process zone was distributed by the variable $p$ that governs the recovery of the residual strength from the delamination tip.

$$
s=s_{i n i} r^{p}, \quad s_{i n i} \text { : initial value of } s, 0 \leq r \leq 1
$$

The distance from the notch to the transverse crack farthest from the notch was defined as the variable $d_{c}$. The alignment of the FBG sensor in this study offers little sensitivity to the splits in the reflection spectrum ${ }^{(16)}$. The length of split $d_{s 1}$ and splitting process zone $d_{s 2}$ were then related to the delamination process zone $d_{d 3}$, since the delamination extends from the tips of the splits ${ }^{(13)}$. Here, we assumed that the split tip coincided with the delamination process zone. A small splitting process zone was also assumed; the residual strength parameter in the splitting process zone was distributed by the variable $p$ as in the delamination process zone.

Substituting the determined distribution of the residual strength parameter into Eq. (2) or (12) provides the strain distribution of the FBG sensor for damage pattern $\boldsymbol{d}$. The following optical analysis can simulate the corresponding reflection spectrum $\tilde{a}_{m}(\boldsymbol{d})$.

\subsection{Procedure}

Figure 6 illustrates the flowchart for the damage identification. Our previous study ${ }^{(16)}$ demonstrated that the spectrum shape was deformed differently by each type of damage. In order to reduce the computation time, we estimated the variables for the damage pattern in the following hierarchical order: (1) sizes of the delamination $\left(d_{d 1}, d_{d 2}, d_{d 3}\right)$, (2) shapes of the delamination $(\alpha, \beta, p)$, and (3) transverse cracks $\left(d_{c}\right)$. The variables in the earlier
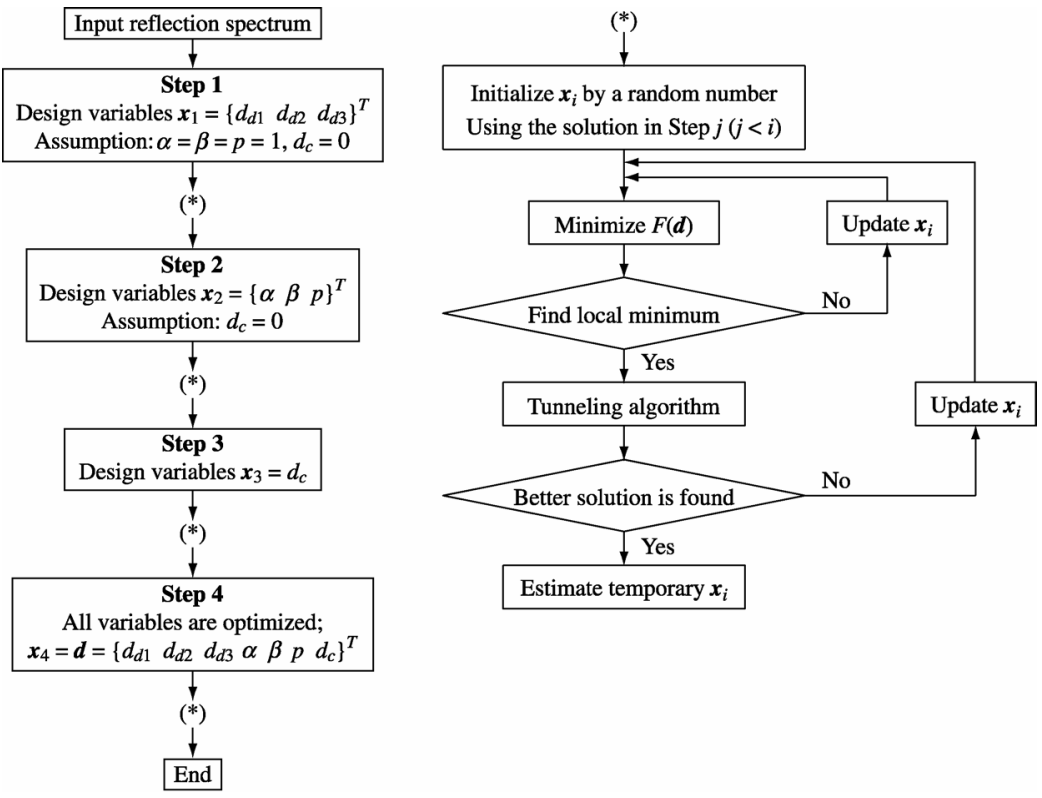

Fig. 6 Flowchart for damage identification. 
estimation had the greater effects on the spectrum shape. Finally, all variables were simultaneously estimated using the solutions of the stepwise estimations.

The initial values of the variables were prepared by a random number in each estimation step. The objective function $F(\boldsymbol{d})$ was minimized by using mathematical programming (the Fletcher-Powell method with the golden-section linear search). The gradient for variable $d_{i}$ was calculated by the finite-difference method in the optimization process.

$$
\frac{\partial F}{\partial d_{i}} \approx \frac{F\left(d_{i}+\Delta d_{i}\right)-F\left(d_{i}\right)}{\Delta d_{i}}
$$

The objective function $F(\boldsymbol{d})$ contains many locally optimal solutions, so we introduced the tunneling algorithm ${ }^{(22)}$ to obtain the globally optimal solution.

\section{Results and discussion}

\subsection{Damage identification for numerical examples}

This section applied the damage identification to the numerical examples. The damage patterns in Fig. 3 were estimated from the corresponding spectrum depicted in Fig. 4 (solid lines). The estimated spectra are also depicted in Fig. 4 by the dashed lines; their shapes are almost identical to those of the input spectra in all cases.

The estimated damage patterns are depicted in Fig. 3 (Estimation). The size and shape of the delamination and the transverse cracks agreed well with the simulation. Variables $d_{d 2}$ (size of the delamination in the transverse direction) and $d_{c}$ (transverse cracks) were particularly well identified. These two variables caused the delamination and the transverse cracks to intersect the FBG sensor; these consequently had larger effects on the spectrum shape than the other variables. This result suggested that the estimation could be improved by embedding the FBG sensor at the position where all design variables (all types of damage) have high sensitivity to the spectrum shape.

This study evaluated the spectrum shape using a Fourier series in the objective function (23). This expression acted as a filter to trivial changes in the spectrum shape. On the other hand, the Fourier coefficients had no physical meaning and might have removed important information on the damage pattern. Such a problem, however, did not occur in this study.

\subsection{Effects of the tunneling algorithm}

The previous section obtained the globally optimal solution for the minimal $F(d)$ using the tunneling algorithm ${ }^{(22)}$. This section clarifies its effect by estimating the damage pattern without this algorithm. The best result among fifty estimations is depicted in Fig. 7, where the input spectrum was the calculation at $0.45 \%$ strain depicted in Fig. 4 (c) by the solid line. The value of the function $F(\boldsymbol{d})$ was $9.56 \times 10^{-3}$, and the splits were underestimated.

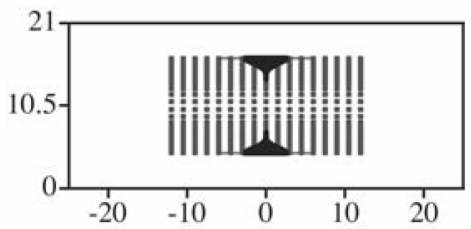

(a) Damage pattern

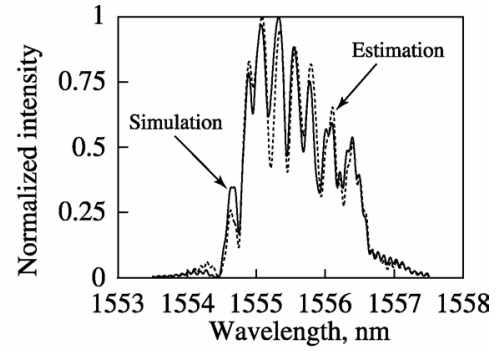

(b) Reflection spectrum

Fig. 7 Estimated results without the tunneling algorithm for simulated results at $0.45 \%$ strain. 


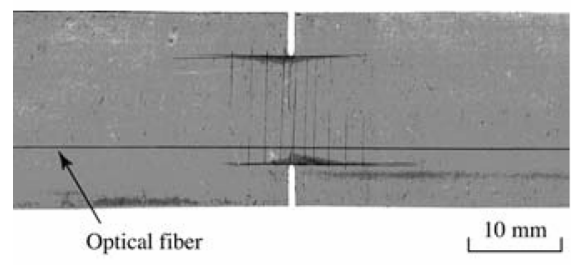

(a) X-ray photograph

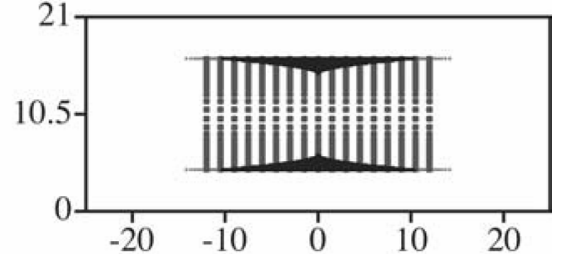

(b) Estimated damage pattern

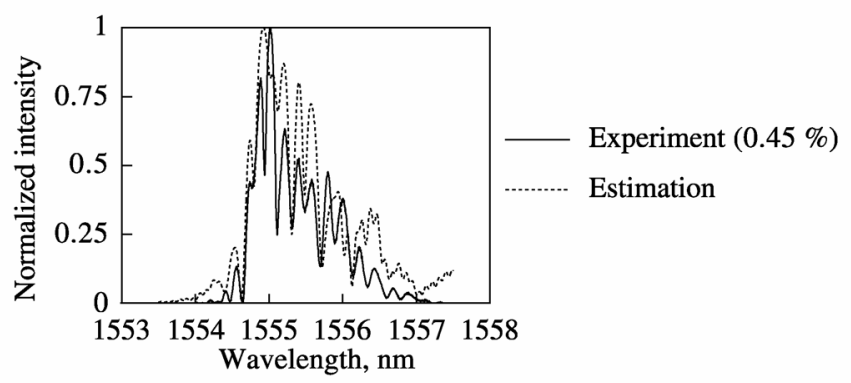

(c) Reflection spectrum

Fig. 8 Damage identification for the experiment result at a $0.45 \%$ strain in a notched CFRP cross-ply laminate.

In contrast, the value $F(\boldsymbol{d})$ with the tunneling algorithm was $4.15 \times 10^{-4}$ for the estimated result in Figs. 3(c) and 4(c). This result confirmed the effect of the tunneling algorithm on improving the estimation.

\subsection{Application to an experiment result}

We demonstrated the damage identification for an experiment result. Figure 8 depicts the experiment result of the observed damage pattern and the measured reflection spectrum at $0.45 \%$ strain for a notched CFRP cross-ply laminate. The damage pattern and reflection spectrum estimated from the measured spectrum are also depicted in Fig. 8; these estimation results agreed well with the experiment results. We thus confirmed that the proposed damage identification technique could be used in practical situations.

\section{Conclusions}

This study proposed and demonstrated an inverse analysis for damage identification in notched composite laminates using the reflection spectrum of an embedded FBG sensor. Variables representing the damage pattern near the notch were estimated through mathematical programming by minimizing the differences of the spectrum shapes between the inputs and the estimations. We applied the developed damage identification to numerical examples and an experiment result for a notched CFRP cross-ply laminate under tension. We summarize our conclusions below.

(1) The estimated damage patterns from the calculated reflection spectra were almost identical to the corresponding simulated damage patterns at several strain levels. These results confirmed the validity of the proposed procedure.

(2) The estimation could be improved by embedding the FBG sensor at the position where the damage pattern has high sensitivity to the spectrum shape.

(3) We confirmed the effects of the tunneling algorithm in improving the accuracy for the damage identification.

(4) The damage pattern estimated from a measured spectrum agreed well with the corresponding damage pattern observed in the experiment. This result confirmed the applicability of the damage identification technique for practical usage. 


\section{References}

(1) Chang, F.K. ed., Structural Health Monitoring 2003, (2003), p. 1, DEStech Publications.

(2) Merzbacher, C.I. et al., Fiber optic sensors in concrete structures: A review, Smart Materials and Structures, Vol. 5, No. 2 (1996), pp. 196-208.

(3) Kageyama, K. et al., Smart marine structures: an approach to the monitoring of ship structures with fiber-optic sensors, Smart Materials and Structures, Vol. 7, No. 4 (1998), pp. 472-478.

(4) Kosaka, T. et al., Strain monitoring of braided composites by using embedded fiber-optic strain sensors, Advanced Composite Materials, Vol. 13, No. 3-4 (2004), pp. 157-170.

(5) Tsuda, H. et al., Impact damage detection in CFRP using fiber Bragg gratings, Smart Materials and Structures, Vol. 13, No. 4 (2004), pp. 719-724.

(6) Tajima, N. et al., Overview of the Japanese smart materials demonstrator program and structures system project, Advanced Composite Materials, Vol. 13, No. 1 (2004), pp. 3-15.

(7) Yari, T. et al., Aircraft structural health monitoring using optical fiber distributed BOTDR sensors, Advanced Composite Materials, Vol. 13, No. 1 (2004), pp. 17-26.

(8) Tsutsui, H. et al., Detection of impact damage of stiffened composite panels using embedded small-diameter optical fibers. Smart Materials and Structures, Vol. 13, No. 6 (2004), pp. 1284-1290.

(9) Hill, K.O., Meltz, G., Fiber Bragg grating technology fundamentals and overview, Journal of Lightwave Technology, Vol. 15, No. 8 (1997), pp. 1263-1276.

(10) Kersey, A.D. et al., Fiber grating sensors, Journal of Lightwave Technology, Vol. 15, No. 8 (1997), pp. 1442-1463.

(11) Okabe, Y. et al., Detection of transverse cracks in CFRP composites using embedded fiber Bragg grating sensors, Smart Materials and Structures, Vol. 9, No .6 (2000), pp. 832-838.

(12) Takeda, S. et al., Delamination detection in CFRP laminates with embedded small-diameter fiber Bragg grating sensors, Composites Part A, Vol. 33, No. 7 (2002), pp. 971-980.

(13) Kortschot, M.T., Beaumont, P.W.R., Damage mechanics of composite materials: Imeasurements of damage and strength, Composites Science and Technology, Vol. 39, No. 4 (1990), pp. 289-301.

(14) Chang, K.Y. et al., Damage tolerance of laminated composites containing an open hole and subjected to tensile loadings, Journal of Composite Materials, Vol. 25, No. 3 (1991), pp. 274-301.

(15) Kamiya, S., Sekine, H., Prediction of the fracture strength of notched continuous fiber-reinforced laminates by interlaminar crack extension analysis, Composites Science and Technology, Vol. 56, No. 1 (1996), pp. 11-21.

(16) Yashiro, S. et al., A new approach to predicting multiple damage states in composite laminates with embedded FBG sensors, Composites Science and Technology, Vol. 65, No. 3-4 (2005), pp. 659-667.

(17) Geubelle, P.H., Baylor, J.S., Impact-induced delamination of composites: a 2D simulation, Composites Part B, Vol. 29, No. 5 (1998), pp. 589-602.

(18) Owen, D.R.J., Hinton, E., Finite Elements in Plasticity, (1980), p. 13, Pineridge Press.

(19) Huang, S. et al., Bragg intragrating structural sensing, Applied Optics, Vol. 34, No. 22 (1995), pp. 5003-5009.

(20) Van Steenkiste, R.J., Springer, G.S., Strain and temperature measurement with fiber optic sensors, (1997), p. 162, Technomic Publication.

(21) Okabe, Y. et al., Application of chirped fiber Bragg grating sensors for identification of crack locations in composites, Composites Part A, Vol. 35, No. 1 (2004), pp. 59-65.

(22) Levy, A.V., Montalvo, A., The tunneling algorithm for the global minimization of functions, SIAM Journal on Scientific Computing, Vol. 6, No. 1 (1985), pp. 15-29. 